Pacific

Journal of

Mathematics

\title{
ON SHRINKING GRADIENT RICCI SOLITONS WITH NONNEGATIVE SECTIONAL CURVATURE
}

\author{
Mingliang CAI
}




\title{
ON SHRINKING GRADIENT RICCI SOLITONS WITH NONNEGATIVE SECTIONAL CURVATURE
}

\author{
Mingliang CAi
}

\begin{abstract}
Perelman proved that an open 3-dimensional shrinking gradient Ricci soliton with bounded nonnegative sectional curvature is a quotient of $S^{2} \times \mathbb{R}$ or $\mathbb{R}^{3}$. We extend this result to higher dimensions with a decay condition on the Ricci tensor.
\end{abstract}

\section{Introduction}

A gradient Ricci soliton is a Riemannian manifold $(M, g)$ together with a smooth function $f$ such that

$$
\text { Ric }+ \text { Hess } f=\lambda g,
$$

where $\lambda$ is a constant. It is called shrinking, steady and expanding when $\lambda>0$, $\lambda=0$ and $\lambda<0$ respectively.

Gradient Ricci solitons are self-similar solutions of Hamilton's Ricci flow and play a vital role in the analysis of singularities of the flow. In dimension 2, Hamilton [1988] completely classified shrinking gradient Ricci solitons with bounded curvature and proved that they are the sphere, the projective space and the Euclidean space with constant curvature. In dimension 3, Ivey [1993] proved that compact shrinking gradient Ricci solitons have positive sectional curvature, and Perelman [2003] proved that shrinking gradient Ricci solitons with bounded nonnegative sectional curvature are quotients of $S^{3}, S^{2} \times \mathbb{R}$ or $\mathbb{R}^{3}$.

In higher dimensions, there have been many results in the last several years. Chen [2009] showed that a complete shrinking gradient Ricci soliton has nonnegative scalar curvature. Ni and Wallach [2008] gave the classification of shrinking gradient Ricci solitons with nonnegative Ricci curvature and zero Weyl tensor. Petersen and Wylie [2010] and independently, Cao, Wang and Zhu [Cao et al. 2011], classified the shrinking gradient Ricci solitons with zero Weyl tensor. Fernández-López and García-Río [2011] considered solitons with harmonic Weyl tensor. In [Petersen and Wylie 2009], several natural curvature conditions are given that characterize gradient Ricci solitons of the flat vector bundle $N \times_{\Gamma} \mathbb{R}^{m}$, where $N$ is an Einstein manifold,

MSC2010: primary 53C25; secondary 53C20, 53C24.

Keywords: shrinking gradient Ricci soliton, rigidity, nonnegative sectional curvature. 
$\Gamma$ acts freely on $N$ and by orthogonal transformations on $\mathbb{R}^{m}$, and $f=\frac{1}{4} d^{2}$ with $d$ being the distance on the flat fiber to the base. In particular, it is shown in [Petersen and Wylie 2009] that a shrinking gradient Ricci soliton is rigid, i.e., of the form $N \times{ }_{\Gamma} \mathbb{R}^{m}$, if the scalar curvature is constant and the sectional curvature of the plane containing $\nabla f$ is nonnegative. As a consequence of a theorem of Böhm and Wilking [2008], the gradient Ricci solitons with positive curvature operators are trivial. In view of this and the aforementioned result of Perelman, one naturally asks to what extend shrinking gradient Ricci solitons with nonnegative sectional curvature are rigid. Our first result in this paper is the rigidity under a decay condition on $\mid D$ Ric $\mid$, extending Perelman's result to higher dimensions. In all theorems we scale the metric so that $\lambda=\frac{1}{2}$.

Theorem 1.1. Let $(M, g, f)$ be a complete noncompact shrinking gradient Ricci soliton with bounded nonnegative sectional curvature. Assume that there exists $\delta>0$ such that

$$
\int_{M} e^{\delta f}|D \mathrm{Ric}| d \operatorname{vol}_{g}<\infty
$$

Then $\left(M^{n}, g\right)$ is isometric to $N \times_{\Gamma} \mathbb{R}^{m}$, where $N$ is a compact Einstein manifold.

This is, to our knowledge, the first rigidity result in high dimensions without assumptions on the Weyl tensor. The potential function $f$ is known to grow quadratically with respect to the distance from a fixed point, so our condition on $D$ Ric says that it decays exponentially. Our proof also works under the assumption that $D$ Ric decays polynomially with a degree depending on other geometric quantities.

The Cheeger-Gromoll soul theorem states that an open manifold with nonnegative sectional curvature is diffeomorphic to a vector bundle over a compact submanifold called a soul. The pull-back metric on the bundle can be highly twisted. However, if there exists a gradient soliton structure on such a bundle, then, by Theorem 1.1, the metric has to be locally trivial, provided that the decay condition is satisfied. The decay condition on $D$ Ric in Theorem 1.1 is imposed in the region where $f$ is large. Our next result deals with the rigidity under a condition on $D$ Ric imposed in the region where $f$ is small.

Theorem 1.2. Let $\left(M^{n}, g, f\right)$ be a complete shrinking gradient Ricci soliton with bounded nonnegative sectional curvature. Assume that the minima of $f$ is a smooth compact nondegenerate critical submanifold and D Ric and $D^{2}$ Ric vanish on the minima. Then $\left(M^{n}, g\right)$ is noncompact and isometric to $N \times_{\Gamma} \mathbb{R}^{m}$, where $N$ is a compact Einstein manifold.

We derive some basic formulas in Section 2, and prove Theorems 1.1 and 1.2 in Sections 3 and 4 respectively. 


\section{Basic formulas}

There are different conventions for the curvature tensor in the literature, so to avoid the confusion, we state ours as follows. The $(3,1)$ tensor $\operatorname{Rm}(X, Y, Z)=$ $\operatorname{Rm}(X, Y) Z$ is defined as

$$
\operatorname{Rm}(X, Y) Z=D_{X} D_{Y} Z-D_{Y} D_{X} X-D_{[X, Y]} Z
$$

and the $(4,0)$ tensor as

$$
\operatorname{Rm}(X, Y, Z, W)=\langle\operatorname{Rm}(X, Y) Z, W\rangle .
$$

We use Ric to denote the Ricci tensor and $R$ the scalar curvature. For a tangent vector $X$ at $p$, we use $\operatorname{Ric}(X)$ to denote the vector such that

$$
\langle\operatorname{Ric}(X), Y\rangle=\operatorname{Ric}(X, Y)
$$

for any vector $Y$ at $p$. For any smooth vector field $V$ and any smooth function $\phi$ on manifold $M$, by $V(\phi)$, we mean $V(\phi)=d \phi(V)=\langle V, \nabla \phi\rangle$. In the remainder of the paper, we will rescale the metric and assume that our gradient Ricci soliton satisfies

$$
\text { Ric }+ \text { Hess } f=\frac{1}{2} g \text {. }
$$

Since the curvature of $(M, g)$ is assumed to be bounded, there exists a flow $\Phi_{t}: M \rightarrow M$ defined for all time with $\Phi_{0}=\mathrm{Id}$ and $\partial \Phi / \partial t=\nabla f$ [Morgan and Tian 2007, p. 207]. For $t \in(\infty, 0)$, define $G(t)=|t| \Phi_{-\ln |t|}^{*} g$. Then $G(-1)=g$ and $G(t)$ satisfies

$$
\operatorname{Ric}(G(t))+\operatorname{Hess} f=\frac{1}{2 \tau} G(t),
$$

where Hess is taken with respect to the metric $G(t)$ and $\tau=|t|=-t$.

In the next lemma, we collect some well-known formulae.

Lemma 2.1. On $(M, G(t))$, we have

(1) $d R=2 \operatorname{Ric}(\nabla f, \cdot)$,

(2) $|\nabla f|^{2}=f / \tau-R+$ constant,

(3) $R / \tau+\langle\nabla f, \nabla R\rangle=\Delta R+2|\mathrm{Ric}|^{2}$,

(4) $\operatorname{div} \operatorname{Rm}(X, Y, Z)=\operatorname{Rm}(\nabla f, X, Y, Z)$,

(5) $D_{X} \operatorname{Ric}(Y, Z)=D_{Y} \operatorname{Ric}(X, Z)-\operatorname{Rm}(X, Y, \nabla f, Z)$,

where $\operatorname{div} \operatorname{Rm}(X, Y, Z)=\operatorname{trace}_{1,2} D \operatorname{Rm}(\cdot, \cdot, X, Y, Z)$.

Proof. The derivations of (1)-(3) can be found in [Hamilton 1995] and (4)-(5) in [Petersen and Wylie 2010]. 
Lemma 2.2. On $(M, g)$, we have

$$
\Delta \mid \text { Ric }\left.\right|^{2}=2 \mid D \text { Ric }\left.\right|^{2}+2 \mid \text { Ric }\left.\right|^{2}+\nabla f\left(\mid \text { Ric }\left.\right|^{2}\right)-4 K_{i j} \lambda_{i} \lambda_{j},
$$

where $\lambda_{i}$ are the eigenvalues of the Ricci tensor and $K_{i j}$ is the sectional curvature of the plane spanned by the eigenvectors belonging to $\lambda_{i}$ and $\lambda_{j}$ respectively.

Proof. This follows from the formula derived in Lemma 2.1 in [Petersen and Wylie 2010]:

$$
\Delta \operatorname{Ric}=D_{\nabla f} \operatorname{Ric}+\operatorname{Ric}-2 \sum_{k=1}^{n} \operatorname{Rm}\left(\cdot, e_{k}, \operatorname{Ric}\left(e_{k}\right), \cdot\right) .
$$

Throughout the computations in the paper, we assume $\left\{e_{1}, \ldots, e_{n}\right\}$ is an orthonormal basis in a neighborhood of a fixed point $x$ with $D_{e_{i}} e_{j}(x)=0$ and further assume that each $e_{i}$ is an eigenvector of Ric at $x$ corresponding to the eigenvalue $\lambda_{i}$. Such a basis always exists. We also use the Einstein summation convention (unless otherwise specified).

Lemma 2.3. On $(M, g)$, we have

$$
\operatorname{div}(\operatorname{Ric}(\nabla R))=\nabla f\left(|\operatorname{Ric}|^{2}\right)+\frac{1}{2}|\nabla R|^{2}-2\langle Z, \nabla f\rangle+|\operatorname{Ric}|^{2}-2 \sum_{i} \lambda_{i}^{3},
$$

where $Z=\operatorname{Ric}\left(e_{i}, e_{j}\right) \operatorname{Rm}\left(\nabla f, e_{i}, e_{j}\right)$.

Proof. The following computations are done at $x$. From Lemma 2.1, we have

$$
\begin{aligned}
D_{e_{i}} \operatorname{Ric}\left(\nabla R, e_{i}\right) & =D_{\nabla R} \operatorname{Ric}\left(e_{i}, e_{i}\right)-\operatorname{Rm}\left(e_{i}, \nabla R, \nabla f, e_{i}\right) \\
& =|\nabla R|^{2}-\operatorname{Ric}(\nabla R, \nabla f)=\frac{1}{2}|\nabla R|^{2} .
\end{aligned}
$$

We then obtain

$$
\begin{aligned}
\operatorname{div}(\operatorname{Ric}(\nabla R)) & =\left\langle D_{e_{i}} \operatorname{Ric}(\nabla R), e_{i}\right\rangle=e_{i} \operatorname{Ric}\left(\nabla R, e_{i}\right) \\
& =D_{e_{i}} \operatorname{Ric}\left(\nabla R, e_{i}\right)+\operatorname{Ric}\left(D_{e_{i}} \nabla R, e_{i}\right) \\
& =\frac{1}{2}|\nabla R|^{2}+\operatorname{Ric}\left(e_{i}, e_{j}\right)\left\langle D_{e_{i}} \nabla R, e_{j}\right\rangle \\
& =\frac{1}{2}|\nabla R|^{2}+2 \operatorname{Ric}\left(e_{i}, e_{j}\right)\left\langle D_{e_{i}} \operatorname{Ric}(\nabla f), e_{j}\right\rangle \\
& =\frac{1}{2}|\nabla R|^{2}+2 \operatorname{Ric}\left(e_{i}, e_{j}\right) e_{i} \operatorname{Ric}\left(\nabla f, e_{j}\right) \\
& =\frac{1}{2}|\nabla R|^{2}+2 \operatorname{Ric}\left(e_{i}, e_{j}\right)\left(D_{e_{i}} \operatorname{Ric}\left(\nabla f, e_{j}\right)+\operatorname{Ric}\left(D_{e_{i}} \nabla f, e_{j}\right)\right) .
\end{aligned}
$$

That is,

$(2-1) \operatorname{div}(\operatorname{Ric}(\nabla R))=\frac{1}{2}|\nabla R|^{2}+2 \operatorname{Ric}\left(e_{i}, e_{j}\right)\left(D_{e_{i}} \operatorname{Ric}\left(\nabla f, e_{j}\right)+\operatorname{Ric}\left(D_{e_{i}} \nabla f, e_{j}\right)\right)$.

From the soliton equation

$$
\text { Ric }+ \text { Hess } f=\frac{1}{2} g \text {, }
$$

it follows that

$$
D_{e_{i}} \nabla f=\frac{1}{2} e_{i}-\operatorname{Ric}\left(e_{i}\right)=\frac{1}{2} e_{i}-\lambda_{i} e_{i},
$$


where we have used the assumption that $e_{i}$ is an eigenvector of Ric at $x$ belonging to the eigenvalue $\lambda_{i}$. Hence,

$$
2 \operatorname{Ric}\left(e_{i}, e_{j}\right) \operatorname{Ric}\left(D_{e_{i}} \nabla f, e_{j}\right)=2\left(\frac{1}{2}-\lambda_{i}\right)\left(\operatorname{Ric}\left(e_{i}, e_{j}\right)\right)^{2}=2 \lambda_{i}^{2}\left(\frac{1}{2}-\lambda_{i}\right) .
$$

Lemma 2.1(5) implies that

$$
D_{e_{i}} \operatorname{Ric}\left(\nabla f, e_{j}\right)=D_{\nabla f} \operatorname{Ric}\left(e_{i}, e_{j}\right)-\operatorname{Rm}\left(e_{i}, \nabla f, \nabla f, e_{j}\right) .
$$

It follows that

$$
\begin{aligned}
2 \operatorname{Ric}\left(e_{i}, e_{j}\right) D_{e_{i}} & \operatorname{Ric}\left(\nabla f, e_{j}\right) \\
= & 2 \operatorname{Ric}\left(e_{i}, e_{j}\right)\left(D_{\nabla f} \operatorname{Ric}\left(e_{i}, e_{j}\right)-\operatorname{Rm}\left(e_{i}, \nabla f, \nabla f, e_{j}\right)\right) \\
= & 2 \operatorname{Ric}\left(e_{i}, e_{j}\right) D_{\nabla f} \operatorname{Ric}\left(e_{i}, e_{j}\right)-2\langle Z, \nabla f\rangle \\
& =\nabla f\left(|\operatorname{Ric}|^{2}\right)-2\langle Z, \nabla f\rangle .
\end{aligned}
$$

Combining (2-2) and (2-3), we obtain that

$2 \operatorname{Ric}\left(e_{i}, e_{j}\right)\left(D_{e_{i}} \operatorname{Ric}\left(\nabla f, e_{j}\right)+\operatorname{Ric}\left(D_{e_{i}} \nabla f, e_{j}\right)\right)$

$$
=\nabla f\left(|\mathrm{Ric}|^{2}\right)-2\langle Z, \nabla f\rangle+2 \lambda_{i}^{2}\left(\frac{1}{2}-\lambda_{i}\right) .
$$

Substituting the above into (2-1) gives

$$
\begin{aligned}
\operatorname{div}(\operatorname{Ric}(\nabla R)) & =\frac{1}{2}|\nabla R|^{2}+\nabla f\left(|\operatorname{Ric}|^{2}\right)-2\langle Z, \nabla f\rangle+2 \lambda_{i}^{2}\left(\frac{1}{2}-\lambda_{i}\right) \\
& =\frac{1}{2}|\nabla R|^{2}+\nabla f\left(|\operatorname{Ric}|^{2}\right)-2\langle Z, \nabla f\rangle+|\operatorname{Ric}|^{2}-2 \sum_{i} \lambda_{i}^{3} .
\end{aligned}
$$

Remark 2.4. We have $\langle Z, \nabla f\rangle \geq 0$ when the sectional curvature of $(M, g)$ is nonnegative. In fact, at $x,\langle Z, \nabla f\rangle=\lambda_{i} \operatorname{Rm}\left(\nabla f, e_{i}, e_{i}, \nabla f\right)$.

The next lemma is a slight variation of Lemma 2.3.

Lemma 2.5. On $(M, g)$, we have $\nabla f\left(|\mathrm{Ric}|^{2}\right)=2\left(\langle Z, \nabla f\rangle+\sum_{i=1}^{n} \lambda_{i}\left(\lambda_{i}-\frac{1}{2}\right)^{2}\right)+\frac{1}{2}\langle\nabla f, \nabla R\rangle-\frac{1}{2}|\nabla R|^{2}-\operatorname{div}\left(D_{\nabla R} \nabla f\right)$.

Proof. It follows from Lemma 2.3 that

$$
\operatorname{div}(\operatorname{Ric}(\nabla R))=\frac{1}{2}|\nabla R|^{2}+\nabla f\left(|\operatorname{Ric}|^{2}\right)-2\langle Z, \nabla f\rangle+|\operatorname{Ric}|^{2}-2 \sum_{i} \lambda_{i}^{3} .
$$

Using Ric $(\nabla R)=\frac{1}{2} \nabla R-D_{\nabla R} \nabla f$ and Lemma 2.1(3), we have

$$
\begin{aligned}
\nabla f\left(|\mathrm{Ric}|^{2}\right)=\frac{R}{2}-2|\mathrm{Ric}|^{2}+2 \sum_{i} \lambda_{i}^{3}+ & 2\langle Z, \nabla f\rangle \\
& +\frac{1}{2}\langle\nabla f, \nabla R\rangle-\frac{1}{2}|\nabla R|^{2}-\operatorname{div}\left(D_{\nabla R} \nabla f\right) .
\end{aligned}
$$

The lemma now follows as $R / 2-2 \mid$ Ric $\left.\right|^{2}+2 \sum_{i} \lambda_{i}^{3}=2 \sum_{i=1}^{n} \lambda_{i}\left(\lambda_{i}-\frac{1}{2}\right)^{2}$. 
Combining Lemmas 2.2 and 2.3 gives the following proposition.

Proposition 2.6. On $(M, g)$,

$$
P=\frac{1}{2} \nabla f\left(|\mathrm{Ric}|^{2}\right)+\frac{1}{2}|\nabla R|^{2}+\operatorname{div}\left(\frac{1}{2} \nabla|\operatorname{Ric}|^{2}-\operatorname{Ric}(\nabla R)\right),
$$

where $P=K_{i j}\left(\lambda_{i}-\lambda_{j}\right)^{2}+|D \mathrm{Ric}|^{2}+2\langle Z, \nabla f\rangle$.

Proof. Lemma 2.2 implies that

$$
-2 K_{i j} \lambda_{i} \lambda_{j}+\mid D \text { Ric }\left.\right|^{2}=-\frac{1}{2} \nabla f\left(|\operatorname{Ric}|^{2}\right)-|\operatorname{Ric}|^{2}+\operatorname{div}\left(\frac{1}{2} \nabla|\operatorname{Ric}|^{2}\right),
$$

while Lemma 2.3 implies that

$$
2 \sum_{i} \lambda_{i}^{3}+2\langle Z, \nabla f\rangle=\nabla f\left(|\operatorname{Ric}|^{2}\right)+|\operatorname{Ric}|^{2}+\frac{1}{2}|\nabla R|^{2}-\operatorname{div}(\operatorname{Ric}(\nabla R)) .
$$

Adding the corresponding sides of the last two equations and noting that $2 \sum_{i} \lambda_{i}^{3}-$ $2 \sum_{i, j} K_{i j} \lambda_{i} \lambda_{j}=\sum_{i, j} K_{i j}\left(\lambda_{i}-\lambda_{j}\right)^{2}$, we obtain Proposition 2.6.

Remark 2.7. Clearly, $P \geq 0$ when the sectional curvature of $(M, g)$ is nonnegative.

The proof of Theorem 1.1 will use an alternative form of Proposition 2.6 in which the term $\mid D$ Ric $\left.\right|^{2}$ is replaced by $|\operatorname{div} \mathrm{Rm}|^{2}$. An integral from of the next lemma is proved in [Cao 2007].

Lemma 2.8. On $(M, g)$,

$$
\mid D \text { Ric }\left.\right|^{2}=|\operatorname{div} \operatorname{Rm}|^{2}+2\langle Z, \nabla f\rangle-\frac{1}{2} \nabla f\left(|\operatorname{Ric}|^{2}\right)+\operatorname{div}\left(\frac{1}{2} \nabla|\operatorname{Ric}|^{2}-2 Z\right) .
$$

Proof. As before, we fix an orthonormal basis, $\left\{e_{1}, \ldots, e_{n}\right\}$, in a neighborhood of a fixed point $x$ and assume that $D_{e_{i}} e_{j}(x)=0$ and that each $e_{i}$ is an eigenvector of Ric at $x$ corresponding to the eigenvalue $\lambda_{i}$. Recall that $Z=\operatorname{Ric}\left(e_{i}, e_{j}\right) \operatorname{Rm}\left(\nabla f, e_{i}, e_{j}\right)$, so at $x$,

$$
\begin{aligned}
\operatorname{div}(Z)= & \left\langle D_{e_{k}} Z, e_{k}\right\rangle=\left\langle D_{e_{k}}\left(\operatorname{Ric}\left(e_{i}, e_{j}\right) \operatorname{Rm}\left(\nabla f, e_{i}, e_{j}\right)\right), e_{k}\right\rangle \\
= & e_{k}\left(\operatorname{Ric}\left(e_{i}, e_{j}\right)\right) \operatorname{Rm}\left(\nabla f, e_{i}, e_{j}, e_{k}\right)+\operatorname{Ric}\left(e_{i}, e_{j}\right)\left\langle D_{e_{k}}\left(\operatorname{Rm}\left(\nabla f, e_{i}, e_{j}\right)\right), e_{k}\right\rangle \\
= & D_{e_{k}} \operatorname{Ric}\left(e_{i}, e_{j}\right) \operatorname{Rm}\left(\nabla f, e_{i}, e_{j}, e_{k}\right)+\operatorname{Ric}\left(e_{i}, e_{j}\right) e_{k}\left(\operatorname{Rm}\left(\nabla f, e_{i}, e_{j}, e_{k}\right)\right) \\
= & D_{e_{k}} \operatorname{Ric}\left(e_{i}, e_{j}\right) \operatorname{div} \operatorname{Rm}\left(e_{i}, e_{j}, e_{k}\right) \\
& \quad+\operatorname{Ric}\left(e_{i}, e_{j}\right)\left(D_{e_{k}} \operatorname{Rm}\left(\nabla f, e_{i}, e_{j}, e_{k}\right)+\operatorname{Rm}\left(D_{e_{k}} \nabla f, e_{i}, e_{j}, e_{k}\right)\right) \\
= & \left(D_{e_{i}} \operatorname{Ric}\left(e_{j}, e_{k}\right)-\operatorname{Rm}\left(e_{k}, e_{i}, \nabla f, e_{j}\right)\right) \operatorname{div} \operatorname{Rm}\left(e_{i}, e_{j}, e_{k}\right) \\
& \quad+\operatorname{Ric}\left(e_{i}, e_{j}\right) \operatorname{div} \operatorname{Rm}\left(e_{j}, e_{i}, \nabla f\right)+\lambda_{i} \operatorname{Rm}\left(\left(\frac{1}{2}-\lambda_{k}\right) e_{k}, e_{i}, e_{i}, e_{k}\right) \\
= & D_{e_{i}} \operatorname{Ric}\left(e_{j}, e_{k}\right) \operatorname{div} \operatorname{Rm}\left(e_{i}, e_{j}, e_{k}\right)+\operatorname{div} \operatorname{Rm}\left(e_{j}, e_{i}, e_{k}\right) \operatorname{div} \operatorname{Rm}\left(e_{i}, e_{j}, e_{k}\right) \\
& \quad+\operatorname{Ric}\left(e_{i}, e_{j}\right) \operatorname{Rm}\left(\nabla f, e_{j}, e_{i}, \nabla f\right)+K_{i j} \lambda_{i}\left(\frac{1}{2}-\lambda_{j}\right) .
\end{aligned}
$$


In the above calculation, we have repeatedly used Lemma 2.1. The lemma now follows from Lemma 2.2 and the following two identities, whose proofs are easy:

$$
\begin{gathered}
D_{e_{i}} \operatorname{Ric}\left(e_{j}, e_{k}\right) \operatorname{div} \operatorname{Rm}\left(e_{i}, e_{j}, e_{k}\right)=0, \\
\operatorname{div} \operatorname{Rm}\left(e_{j}, e_{i}, e_{k}\right) \operatorname{div} \operatorname{Rm}\left(e_{i}, e_{j}, e_{k}\right)=\frac{1}{2}|\operatorname{div} \operatorname{Rm}|^{2} .
\end{gathered}
$$

Lemma 2.8, together with Proposition 2.6, implies the following:

Lemma 2.9. On $(M, g)$,

$$
Q=\nabla f\left(|\operatorname{Ric}|^{2}\right)+\frac{1}{2}|\nabla R|^{2}+\operatorname{div}(2 Z-\operatorname{Ric}(\nabla R)),
$$

where $Q=K_{i j}\left(\lambda_{i}-\lambda_{j}\right)^{2}+|\operatorname{div} \mathrm{Rm}|^{2}+4\langle Z, \nabla f\rangle$.

Remark 2.10. We note that $Q \geq 0$ when the sectional curvature of $(M, g)$ is nonnegative.

The next lemma deals with the term $\nabla f\left(\mid\right.$ Ric $\left.\left.\right|^{2}\right)$ in Lemma 2.9.

Lemma 2.11. On $(M, g)$,

$$
\begin{aligned}
\nabla f\left(|\mathrm{Ric}|^{2}\right)=\frac{1}{2}|\nabla R|^{2}+\frac{1}{2}\langle\nabla f, \nabla R\rangle+\frac{1}{2} \nabla & f(\langle\nabla f, \nabla R\rangle) \\
& +\operatorname{div}\left(D_{\nabla R} \nabla f-\frac{1}{2} \nabla\langle\nabla f, \nabla R\rangle\right) .
\end{aligned}
$$

Proof. It follows from Lemma 2.1(1) and (3) that

$$
\frac{1}{2} \nabla f(\Delta R)=-\nabla f\left(|\mathrm{Ric}|^{2}\right)+\frac{1}{2}\langle\nabla f, \nabla R\rangle+\frac{1}{2} \nabla f(\langle\nabla f, \nabla R\rangle) .
$$

The Bochner-Weitzenböck formula implies that

$$
\begin{aligned}
\operatorname{div}\left(\frac{1}{2} \nabla\langle\nabla f, \nabla R\rangle\right) & =\frac{1}{2} \Delta\langle\nabla f, \nabla R\rangle \\
& =\langle\text { Hess } f, \text { Hess } R\rangle+\frac{1}{2} \nabla f(\Delta R)+\frac{1}{2} \nabla R(\Delta f)+\operatorname{Ric}(\nabla f, \nabla R) \\
& =\langle\text { Hess } f, \text { Hess } R\rangle+\frac{1}{2} \nabla f(\Delta R)+\frac{1}{2} \nabla R\left(\frac{n}{2}-R\right)+\frac{1}{2}|\nabla R|^{2} \\
& =\langle\text { Hess } f, \text { Hess } R\rangle+\frac{1}{2} \nabla f(\Delta R) .
\end{aligned}
$$

But,

$$
\begin{aligned}
\operatorname{div}\left(D_{\nabla R} \nabla f\right) & =\left\langle D_{e_{i}} D_{\nabla R} \nabla f, e_{i}\right\rangle=e_{i}\left\langle D_{\nabla R} \nabla f, e_{i}\right\rangle=e_{i}\left\langle D_{e_{i}} \nabla f, \nabla R\right\rangle \\
& =\left\langle D_{e_{i}}\left(\frac{1}{2} e_{i}-\operatorname{Ric}\left(e_{i}\right)\right), \nabla R\right\rangle+\langle\operatorname{Hess} f, \text { Hess } R\rangle \\
& =-D_{e_{i}} \operatorname{Ric}\left(e_{i}, \nabla R\right)+\langle\text { Hess } f, \text { Hess } R\rangle \\
& =-\frac{1}{2}|\nabla R|^{2}+\langle\operatorname{Hess} f, \text { Hess } R\rangle .
\end{aligned}
$$

The lemma follows.

We now have the following proposition which will be used in the proof of Theorem 1.1. 
Proposition 2.12. On $(M, g)$,

$$
\begin{aligned}
Q=|\nabla R|^{2}+\frac{1}{2}\langle\nabla f, \nabla R\rangle+\frac{1}{2} \nabla & f(\langle\nabla f, \nabla R\rangle) \\
& +\operatorname{div}\left(2 Z-\operatorname{Ric}(\nabla R)+D_{\nabla R} \nabla f-\frac{1}{2} \nabla\langle\nabla f, \nabla R\rangle\right) .
\end{aligned}
$$

Proof. This is merely a consequence of Lemmas 2.9 and 2.11.

\section{Proof of Theorem 1.1}

We will use $\phi$ to denote a real-valued nonnegative $C^{4}$ function on $\mathbb{R}$ and write $\phi \circ f$ as $\phi(f)$. We will show that $R$ is a constant function and then appeal to [Petersen and Wylie 2009] to complete the proof. We begin with the following proposition.

Proposition 3.1. On $(M, g)$,

$$
\begin{array}{r}
\phi(f) Q=\frac{1}{2}\langle\nabla f, \nabla R\rangle\left(\left(\phi-\phi^{\prime}\right)(f)-\left(\phi+\phi^{\prime}\right)(f) \Delta f-\left(\phi^{\prime \prime}+\phi^{\prime}\right)(f)|\nabla f|^{2}\right) \\
+\left(\phi+\phi^{\prime}\right)(f)|\nabla R|^{2}-2 \phi^{\prime}\langle Z, \nabla f\rangle+\operatorname{div}(X),
\end{array}
$$

where

$$
X=\frac{1}{2}\langle\nabla f, \nabla R\rangle\left(\phi^{\prime}+\phi\right)(f) \nabla f+\phi(f)\left(2 Z-\operatorname{Ric}(\nabla R)+D_{\nabla R} \nabla f-\frac{1}{2} \nabla\langle\nabla f, \nabla R\rangle\right) .
$$

Proof. We multiply each side of the equation in Proposition 2.12 by $\phi(f)$ to get

$$
\begin{aligned}
\phi(f) Q= & \phi(f)|\nabla R|^{2}+\frac{\phi(f)}{2}\langle\nabla f, \nabla R\rangle+\frac{\phi(f)}{2} \nabla f(\langle\nabla f, \nabla R\rangle) \\
& -\phi^{\prime}(f)\left\langle 2 Z-\operatorname{Ric}(\nabla R)+D_{\nabla R} \nabla f-\frac{1}{2} \nabla\langle\nabla f, \nabla R\rangle, \nabla f\right\rangle \\
& +\operatorname{div}\left(\phi(f)\left(2 Z-\operatorname{Ric}(\nabla R)+D_{\nabla R} \nabla f-\frac{1}{2} \nabla\langle\nabla f, \nabla R\rangle\right)\right) .
\end{aligned}
$$

It follows from the soliton equation and Lemma 2.1(1) that

$$
\begin{aligned}
\left\langle-\operatorname{Ric}(\nabla R)+D_{\nabla R} \nabla f, \nabla f\right\rangle & =\left\langle\frac{1}{2} \nabla R-2 \operatorname{Ric}(\nabla R), \nabla f\right\rangle \\
& =\frac{1}{2}\langle\nabla f, \nabla R\rangle-|\nabla R|^{2} .
\end{aligned}
$$

We thus obtain

$$
\begin{aligned}
\phi(f) Q= & \left(\phi+\phi^{\prime}\right)(f)|\nabla R|^{2}+\frac{\phi-\phi^{\prime}}{2}(f)\langle\nabla f, \nabla R\rangle \\
- & 2 \phi^{\prime}\langle Z, \nabla f\rangle+\frac{\phi+\phi^{\prime}}{2}(f) \nabla f(\langle\nabla f, \nabla R\rangle) \\
& +\operatorname{div}\left(\phi(f)\left(2 Z-\operatorname{Ric}(\nabla R)+D_{\nabla R} \nabla f-\frac{1}{2} \nabla\langle\nabla f, \nabla R\rangle\right)\right) .
\end{aligned}
$$

Now, we observe that

$$
\begin{aligned}
\left(\phi+\phi^{\prime}\right)(f) \nabla f(\langle\nabla f, \nabla R\rangle) & =\left\langle\nabla\langle\nabla f, \nabla R\rangle,\left(\phi^{\prime}+\phi\right)(f) \nabla f\right\rangle \\
& =-\langle\nabla f, \nabla R\rangle\left(\left(\phi^{\prime}+\phi\right)(f) \Delta f+\left(\phi^{\prime \prime}+\phi^{\prime}\right)(f)|\nabla f|^{2}\right) \\
& +\operatorname{div}\left(\langle\nabla f, \nabla R\rangle\left(\phi^{\prime}+\phi\right)(f) \nabla f\right) .
\end{aligned}
$$


Substituting the above into (3-2), we obtain (3-1). Proposition 3.1 is thus proved.

The idea now is to choose an appropriate function $\phi$ and integrate (3-1) over $M$. The divergence term, after integration, vanishes because of the fall-off condition we impose. The right-hand side will then be nonpositive while the left is always nonnegative, and consequently, $R$ is a constant. Theorem 1.1 follows from [Petersen and Wylie 2009].

Proof of Theorem 1.1. We normalize $f$ by adding a constant so that Lemma 2.1(2) takes the form $|\nabla f|^{2}=f-R$. Since $R \geq 0$, we always have $|\nabla f|^{2} \leq f$. On the other hand, since $R$ is assumed to be bounded and $f$ grows quadratically with respect to the distance from a fixed point [Cao and Zhou 2010; Naber 2006], we have $|\nabla f|^{2} \geq \frac{1}{2} f$, when $f$ is sufficiently large. Thus, there exists $T>2$ so that when $f \geq T$,

$$
\frac{1}{2} f \leq|\nabla f|^{2} \leq f .
$$

Fix $0<\eta<\delta$ and define $\phi: \mathbb{R} \rightarrow \mathbb{R}$ by $\phi(t)=0$ for $t \leq T$, and $\phi(t)=(t-T)^{k} e^{\eta t}$ for $t \geq T$, where $k$ is a sufficiently large number to be determined. Throughout this section, we will use this $\phi$ in (3-1). By our fall-off assumption, there exists a sequence $t_{i} \rightarrow \infty$ such that

$$
\int_{f=t_{i}} e^{\delta f} \frac{1}{|\nabla f|} \mid D \text { Ric } \mid \rightarrow 0, \quad \text { as } i \rightarrow \infty
$$

From this, we now deduce that

$$
\int_{f \leq t_{i}} \operatorname{div}(X)=\int_{f=t_{i}} \frac{\langle X, \nabla f\rangle}{|\nabla f|} \rightarrow 0, \quad \text { as } i \rightarrow \infty .
$$

To this end, we look at each of the five terms in $X$ and denote by $X_{i}$ the $i$-th term. Then, when $f>T$,

$$
\frac{\left|\left\langle X_{1}, \nabla f\right\rangle\right|}{|\nabla f|}=\frac{1}{2}|\langle\nabla f, \nabla R\rangle|\left(\phi^{\prime}+\phi\right)(f)|\nabla f| \leq C_{1} f^{k+1} e^{\eta f}|\nabla R|,
$$

where $C_{1}$ is a constant depending only on $k$ and $\eta$. Now by the Cauchy-Schwarz inequality,

$$
|D \operatorname{Ric}|^{2}=\sum_{i, j, k}\left(D_{e_{i}} \operatorname{Ric}\left(e_{j}, e_{k}\right)\right)^{2} \geq \frac{1}{n} \sum_{i}\left(\sum_{j} D_{e_{i}} \operatorname{Ric}\left(e_{j}, e_{j}\right)\right)^{2}=\frac{1}{n}|\nabla R|^{2} .
$$

Thus,

$$
|\nabla R| \leq \sqrt{n} \mid D \text { Ric } \mid
$$

Hence,

$$
\frac{\left|\left\langle X_{1}, \nabla f\right\rangle\right|}{|\nabla f|} \leq C_{1} \sqrt{n} f^{k+1} e^{\eta f}|D \mathrm{Ric}| .
$$


Integrating the above over $\left\{f=t_{i}\right\}$ and noting that

$$
C_{1} \sqrt{n} f^{k+1} e^{\eta f}|D \mathrm{Ric}| \leq e^{\delta f} \frac{|D \mathrm{Ric}|}{|\nabla f|}
$$

when $f$ is sufficiently large, we conclude that

$$
\int_{f=t_{i}} \frac{\left|\left\langle X_{1}, \nabla f\right\rangle\right|}{|\nabla f|} \rightarrow 0, \quad \text { as } i \rightarrow \infty .
$$

Now note that $\left\langle X_{2}, \nabla f\right\rangle=2 \phi\langle Z, \nabla f\rangle=2 \phi \sum_{i} \lambda_{i} \operatorname{Rm}\left(\nabla f, e_{i}, e_{i}, \nabla f\right)$. Since Ric is assumed to be bounded and since the sectional curvature is nonnegative,

$$
\frac{\left|\left\langle X_{2}, \nabla f\right\rangle\right|}{|\nabla f|} \leq C_{2} f^{k-1 / 2} e^{\eta f} \operatorname{Ric}(\nabla f, \nabla f)=C_{2} f^{k-1 / 2} e^{\eta f} \frac{1}{2}\langle\nabla f, \nabla R\rangle,
$$

where $C_{2}$ is a constant dependent only on the bound of Ric, and the last equality follows from Lemma 2.1. Hence, when $f$ is sufficiently large,

$$
\frac{\left|\left\langle X_{2}, \nabla f\right\rangle\right|}{|\nabla f|} \leq \frac{1}{2} C_{2} f^{k} e^{\eta f}|\nabla R| \leq e^{\delta f} \frac{\mid D \text { Ric } \mid}{|\nabla f|} .
$$

It then follows that

$$
\int_{f=t_{i}} \frac{\left|\left\langle X_{2}, \nabla f\right\rangle\right|}{|\nabla f|} \rightarrow 0, \quad \text { as } i \rightarrow \infty .
$$

The arguments for the other $X_{i}$ are similar; we will skip $X_{3}$ and $X_{4}$. Now look at $X_{5}$. Repeatedly using Lemma 2.1(2), we see that

$$
\begin{aligned}
\left\langle X_{5}, \nabla f\right\rangle & =-\frac{1}{2} \phi \nabla f(\langle\nabla f, \nabla R\rangle)=-\phi \nabla f(\operatorname{Ric}(\nabla f, \nabla f)) \\
& =-\phi\left(D_{\nabla f} \operatorname{Ric}(\nabla f, \nabla f)+2 \operatorname{Ric}\left(D_{\nabla f} \nabla f, \nabla f\right)\right) \\
& =-\phi\left(D_{\nabla f} \operatorname{Ric}(\nabla f, \nabla f)+\operatorname{Ric}(\nabla f-\nabla R, \nabla f)\right) \\
& =-\phi\left(D_{\nabla f} \operatorname{Ric}(\nabla f, \nabla f)+\frac{1}{2}\langle\nabla f, \nabla R\rangle-\operatorname{Ric}(\nabla R, \nabla f)\right) .
\end{aligned}
$$

Since $|\nabla R|$ can be bounded by $\mid D$ Ric $\mid$, we have $\left|\left\langle X_{5}, \nabla f\right\rangle\right| \leq C_{5} e^{\eta f} f^{k+3} \mid D$ Ric $\mid$. Equation (3-4) then follows.

To simplify notations, we put

$$
\begin{aligned}
F=\frac{1}{2}\langle\nabla f, \nabla R\rangle\left(\left(\phi-\phi^{\prime}\right)(f)-\left(\phi+\phi^{\prime}\right)(f) \Delta\right. & \left.f-\left(\phi^{\prime \prime}+\phi^{\prime}\right)(f)|\nabla f|^{2}\right) \\
& +\left(\phi+\phi^{\prime}\right)(f)|\nabla R|^{2}-2 \phi^{\prime}\langle Z, \nabla f\rangle .
\end{aligned}
$$

Then,

$$
\phi(f) Q=F+\operatorname{div}(X) .
$$

It follows easily from the arguments in the proof of (3-4) that $\int_{M} F d \mathrm{vol}_{g}<\infty$. We thus have

$$
\int_{M} \phi(f) Q=\int_{M} F .
$$


We now show that $\int_{M} F d \operatorname{vol}_{g} \leq 0$. First, we note that $-\Delta f=R-n / 2 \leq \Lambda$, where $\Lambda$ is an upper bound of $R$; hence $-\left(\phi+\phi^{\prime}\right)(f) \Delta f \leq \Lambda\left(\phi+\phi^{\prime}\right)$, as $\phi$ and $\phi^{\prime}$ are both nonnegative. Next, we observe that, by Lemma 2.1,

$$
|\nabla R|^{2}=2 \operatorname{Ric}(\nabla f, \nabla R)=2 \sum_{i} \lambda_{i} e_{i}(f) e_{i}(R)
$$

and $e_{i}(R)=\left\langle\nabla R, e_{i}\right\rangle=2 \operatorname{Ric}\left(\nabla f, e_{i}\right)=2 \lambda_{i} e_{i}(f)$. So for each $i, e_{i}(f) e_{i}(R) \geq 0$. Hence $|\nabla R|^{2} \leq 2 \Lambda\langle\nabla f, \nabla R\rangle$. Finally, we recall that $\langle Z, \nabla f\rangle \geq 0$ (Remark 2.4). We thus conclude, from (3-3), that

$$
F \leq \frac{1}{2}\langle\nabla f, \nabla R\rangle F_{1},
$$

where

$$
F_{1}=\left(\phi-\phi^{\prime}\right)(f)+\Lambda\left(\phi+\phi^{\prime}\right)(f)+4 \Lambda\left(\phi+\phi^{\prime}\right)-\frac{1}{2} f\left(\phi^{\prime \prime}+\phi^{\prime}\right)(f) .
$$

It follows from (3-5) and (3-6) that

$$
\int_{M} \phi(f) Q \leq \frac{1}{2} \int_{M}\langle\nabla f, \nabla R\rangle F_{1} .
$$

A direct computation leads to

$$
\begin{aligned}
F_{1}= & \left(\phi-\phi^{\prime}\right)(t)+\Lambda\left(\phi+\phi^{\prime}\right)(t)+4 \Lambda\left(\phi+\phi^{\prime}\right)(t)-\frac{1}{2} t\left(\phi^{\prime \prime}+\phi^{\prime}\right)(t) \\
=- & \frac{1}{2} \delta(1+\delta)(t-T)^{k+1} e^{\delta t}-\left(\frac{1}{2}(1+2 \delta) k-5(1+\delta) \Lambda-1+\frac{T-2}{2} \delta\right)(t-T)^{k} e^{\delta t} \\
& \quad-k\left(\frac{1}{2}(k-1)-5 \Lambda+\frac{1}{2} T+\right) 1(t-T)^{k-1} e^{\delta t}-\frac{1}{2} T \phi^{\prime \prime} .
\end{aligned}
$$

If we choose $k>10 \Lambda+2$, the above expression will clearly be negative for $t>T$. We have therefore shown that $F_{1} \leq 0$ everywhere and $F_{1}<0$ where $f>T$. Since $Q \geq 0$ (Remark 2.10) and $\langle\nabla f, \nabla R\rangle=2 \operatorname{Ric}(\nabla f, \nabla f) \geq 0$ (Lemma 2.1), we conclude from (3-7) that $\langle\nabla f, \nabla R\rangle=0$ in the region $\{f>T\}$. But as we noted earlier in the proof, $|\nabla R|^{2} \leq 2 \Lambda\langle\nabla f, \nabla R\rangle$. Hence $\nabla R=0$ in the region $\{f>T\}$. The analyticity of the metric [Bando 1987; Kotschwar 2013] then implies that $R$ is a constant function. Theorem 1.1 then follows from [Petersen and Wylie 2009].

\section{Proof of Theorem 1.2}

We first show that the Ricci tensor has a zero eigenvalue at any point $p$ in $C$, then show that the soliton splits in a neighborhood of $p$, which, in turn, implies that the scalar curvature is a constant.

Let $C$ be the critical manifold of minima of $f$. Since $C$ is assumed to be nondegenerate, the Morse-Bott lemma implies that for any point $p \in C$, there exists an open neighborhood $U$ of $p$ and a diffeomorphism $\phi: U \rightarrow \mathbb{R}^{n}$ such that $\phi(U \cap C)=\left\{\left(0, \ldots, 0, x_{m+1}, \ldots, x_{n}\right)\right\}, \phi(p)=0$ and $f \circ \phi^{-1}\left(x_{1}, \ldots, x_{n}\right)=$ $c+\frac{1}{4}\left(x_{1}^{2}+\cdots+x_{m}^{2}\right)$. 
In what follows in this section, unless specified otherwise, the range for the Greek letters $\alpha, \beta, \ldots$ is 1 to $m$ while that for the Latin letters $i, j, \ldots$ is $m+1$ to $n$.

We observe that we may assume that for all $\alpha$ and $i, g^{\alpha i}(p)=0$. In fact, by making a change of variables, $y_{\alpha}=x_{\alpha}$ and $y_{i}=x_{i}-\sum_{\beta=1}^{m} g^{i \beta}(p) x_{\beta}$, we see that in the new coordinates, at $p, g^{\alpha i}=\left\langle\nabla y_{\alpha}, \nabla y_{i}\right\rangle=0$ for $\alpha$ and $i$. Moreover, $f\left(y_{1}, \ldots, y_{m}, y_{m+1}, \ldots, y_{n}\right)=c+\frac{1}{4}\left(y_{1}^{2}+\cdots+y_{m}^{2}\right)$. From now on, we assume in the original coordinates $\left(x_{1}, \ldots, x_{n}\right)$ that $g^{\alpha i}(p)=0$ for all $\alpha$ and $i$. As a consequence, we also have $g_{\alpha i}(p)=0$.

Next lemma computes the Ricci tensor at $p$.

Lemma 4.1. At $p$, we have

$$
\begin{aligned}
\operatorname{Ric}(p)\left(\frac{\partial}{\partial x_{\alpha}}, \frac{\partial}{\partial x_{\beta}}\right)= & \frac{1}{2}\left(g_{\alpha \beta}(p)-\delta_{\alpha \beta}\right), \quad \operatorname{Ric}(p)\left(\frac{\partial}{\partial x_{i}}, \frac{\partial}{\partial x_{j}}\right)=\frac{1}{2} g_{i j}, \\
& \operatorname{Ric}(p)\left(\frac{\partial}{\partial x_{\alpha}}, \frac{\partial}{\partial x_{i}}\right)=0 .
\end{aligned}
$$

Proof. Since

$$
\nabla f=\frac{1}{2} g^{\alpha \beta} x_{\alpha} \frac{\partial}{\partial x_{\beta}}+\frac{1}{2} g^{\alpha i} x_{\alpha} \frac{\partial}{\partial x_{i}},
$$

we have at $p$,

$$
\begin{gathered}
\operatorname{Hess}(f)(p)\left(\frac{\partial}{\partial x_{\alpha}}, \frac{\partial}{\partial x_{\beta}}\right)=\frac{1}{2} \delta_{\alpha \beta}, \\
\operatorname{Hess}(f)(p)\left(\frac{\partial}{\partial x_{\alpha}}, \frac{\partial}{\partial x_{i}}\right)=\operatorname{Hess}(f)(p)\left(\frac{\partial}{\partial x_{i}}, \frac{\partial}{\partial x_{j}}\right)=0 .
\end{gathered}
$$

The lemma follows from the soliton equation.

Let $\mu_{\gamma}^{-1}(\gamma=1, \ldots, m)$ denote the eigenvalues of the positive definite symmetric matrix $g_{\alpha \beta}(p)$. Then there exists $\left(v_{1 \gamma}, \ldots, v_{m \gamma}\right) \neq 0$ such that $\sum_{\beta} g_{\alpha \beta}(p) v_{\beta \gamma}=$ $\mu_{\gamma}^{-1} v_{\alpha \gamma}$. Let $v_{\gamma}=\sum_{\alpha} v_{\alpha \gamma}\left(\partial / \partial x_{\alpha}\right)$. The first part of Lemma 4.1 implies that

$$
\begin{aligned}
\operatorname{Ric}(p)\left(v_{\gamma}, v_{\gamma}\right) & =\sum_{\alpha, \beta} v_{\alpha \gamma} v_{\beta \gamma} \operatorname{Ric}(p)\left(\frac{\partial}{\partial x_{\alpha}}, \frac{\partial}{\partial x_{\beta}}\right) \\
& =\frac{1}{2}\left(\mu_{\gamma}^{-1}-1\right) \sum_{\alpha}\left(v_{\alpha \gamma}\right)^{2} \\
& =\frac{1}{2}\left(\mu_{\gamma}^{-1}-1\right) \mu_{\gamma} g(p)\left(v_{\gamma}, v_{\gamma}\right) \\
& =\frac{1}{2}\left(1-\mu_{\gamma}\right) g(p)\left(v_{\gamma}, v_{\gamma}\right) .
\end{aligned}
$$

We conclude from this and the rest of Lemma 4.1 that the eigenvalues of the Ricci tensor at $p$ are $\left(1-\mu_{\alpha}\right) / 2$, with $\alpha=1, \ldots, m$, and $\frac{1}{2}$ with multiplicity $n-m$. Since the Ricci tensor is assumed to be semipositive definite, $\mu_{\alpha} \leq 1$ for each $\alpha$. Of course, $\mu_{\alpha}>0$. Our goal is to show that $\mu_{\alpha}=1$. 
Now assume $\left\{e_{1}, \ldots, e_{n}\right\}$ is an orthonormal basis in a neighborhood of a fixed point $p \in C$ with $D_{e_{i}} e_{j}(p)=0$ for $1 \leq i, j \leq n$. We may assume that each $e_{\alpha}$ is an eigenvector of Ric at $p$ corresponding to the eigenvalue $\left(1-\mu_{\alpha}\right) / 2$ for $1 \leq \alpha \leq m$ and $e_{i}$ an eigenvector corresponding to $\frac{1}{2}$ for $m+1 \leq i \leq n$.

By our assumption, $D$ Ric $=D^{2}$ Ric $=0$ at $p$. Hence, for each $1 \leq s \leq n$, in the neighborhood of $p$,

$$
\operatorname{Ric}\left(e_{s}, e_{s}\right)=r_{s}+\sum_{i, j, k=1}^{n} r_{s i j k} x_{i} x_{j} x_{k}+\text { higher-order terms, }
$$

where $r_{s}$ and $r_{s i j k}$ are constants. We make the following observation.

Lemma 4.2. Given that $K_{s \alpha}$ is the sectional curvature of the section spanned by $e_{s}$ and $e_{\alpha}$, we have

$r_{\alpha}=\frac{1-\mu_{\alpha}}{2}, \quad \alpha=1, \ldots, m, \quad r_{i}=\frac{1}{2}, \quad i=m+1, \ldots, n, \quad \sum_{\alpha=1}^{m} K_{s \alpha} \mu_{\alpha}=0$,

Proof. We only need to prove the second line. At $p$,

$$
(\Delta \operatorname{Ric})\left(e_{s}, e_{s}\right)=\Delta\left(\operatorname{Ric}\left(e_{s}, e_{s}\right)\right)=0 .
$$

On the other hand, we have $\Delta \operatorname{Ric}=D_{\nabla f} \operatorname{Ric}+\operatorname{Ric}-2 \sum_{l=1}^{n} \operatorname{Rm}\left(\cdot, e_{l}, \operatorname{Ric}\left(e_{l}\right), \cdot\right)$ (Lemma 2.1 in [Petersen and Wylie 2010], see also the proof of Lemma 2.2). Hence,

$$
\begin{aligned}
0 & =\operatorname{Ric}\left(e_{s}, e_{s}\right)-2 \sum_{l=1}^{n} \operatorname{Rm}\left(e_{s}, e_{l}, \operatorname{Ric}\left(e_{l}\right), e_{s}\right) \\
& =r_{s}-2 \sum_{\alpha=1}^{m} \operatorname{Rm}\left(e_{s}, e_{\alpha}, \operatorname{Ric}\left(e_{\alpha}\right), e_{s}\right)-2 \sum_{i=m+1}^{n} \operatorname{Rm}\left(e_{s}, e_{i}, \operatorname{Ric}\left(e_{i}\right), e_{s}\right) \\
& =r_{s}-\sum_{\alpha=1}^{m}\left(1-\mu_{\alpha}\right) \operatorname{Rm}\left(e_{s}, e_{\alpha}, e_{\alpha}, e_{s}\right)-\sum_{i=m+1}^{n} \operatorname{Rm}\left(e_{s}, e_{i}, e_{i}, e_{s}\right) \\
& =\sum_{\alpha=1}^{m} K_{s \alpha} \mu_{\alpha} .
\end{aligned}
$$

We are now in a position to prove Theorem 1.2.

Proof of Theorem 1.2. It follows from Lemma 4.2 and the assumption of nonnegative sectional curvature that $K_{s \alpha}(p)=0$ for all $1 \leq s \leq n$. So, $\operatorname{Ric}(p)$ vanishes on the subspace spanned by $\left\{\partial / \partial x_{\alpha} \mid \alpha=1, \ldots, m\right\}$.

We first prove that a neighborhood of $p$ splits isometrically as $U \times V$, where $U$ is at least $m$-dimensional and Ric $\equiv 0$ on $U$. We have shown that $\operatorname{Ric}_{\alpha \beta}(p)=0$. The rest of the argument is along the lines of the proof of Lemma 8.2 in [Hamilton 
1986] and that of Corollary 2.1 in [Ni and Tam 2003]. Denote by $K(x, t)$ the null space of $\operatorname{Ric}(x, t)$, i.e.,

$$
K(x, t)=\left\{w \in T_{x} M \mid \operatorname{Ric}(x, t)(w)=0\right\} .
$$

Let $w_{0} \in K(p,-1)$ and $\gamma(s)$ a smooth curve starting from $p$. Parallel translating $w_{0}$ along $\gamma$ gives a vector field $w$ along $\gamma$. Denote the extension of $w$ to a neighborhood of $\gamma$ still by $w$. Now we project $w$ onto $K(x, t)$ to get a vector field $v(x, t)$. Then $v(\gamma(s), t) \in K(\gamma(s), t)$. We first show that $D_{\gamma^{\prime}} v$ is also in $K(\gamma(s), t)$. We fix an orthonormal basis in $g(t),\left\{e_{1}, \ldots, e_{n}\right\}$, in a neighborhood of a fixed point $\gamma(s)$ and assume that $e_{i}(\gamma(s))$ are the eigenvectors of Ric. For simplicity, we denote $e_{i}(\gamma(s))$ by $e_{i}(s)$. Since $\operatorname{Ric}(v)=0$, we have $((\partial / \partial t) \operatorname{Ric})(v, v)=0$. The evolution equation for Ricci tensor then implies that at $\gamma(s)$,

$$
(\Delta \operatorname{Ric})(v, v)-2\langle\operatorname{Ric}(v), \operatorname{Ric}(v)\rangle+2 \operatorname{Ric}\left(e_{i}, e_{i}\right) K\left(e_{i}, v\right)=0,
$$

where the repeated indices are being summed over. Since the sectional curvature $K\left(e_{i}, v\right)$ is nonnegative and since $\operatorname{Ric}(v)=0$, we deduce that $(\Delta \operatorname{Ric})(v, v) \leq 0$. Direct computations give

$$
\begin{aligned}
(\Delta \operatorname{Ric})(v, v)=\Delta(\operatorname{Ric}(v, v))-4 e_{i}\left(\operatorname{Ric}\left(v, D_{e_{i}} v\right)\right)+2 \operatorname{Ric}\left(v, D_{e_{i}} D_{e_{i}} v\right) \\
+2 \operatorname{Ric}\left(v, D_{D_{e_{i}} e_{i}} v\right)+2 \operatorname{Ric}\left(D_{e_{i}} v, D_{e_{i}} v\right) .
\end{aligned}
$$

Using $(\Delta \operatorname{Ric})(v, v) \leq 0$ and $\operatorname{Ric}(v)=0$, we obtain $\operatorname{Ric}\left(D_{e_{i}} v, D_{e_{i}} v\right) \leq 0$. Since Ric is positive semidefinite, we conclude that $\operatorname{Ric}\left(D_{e_{i}} v\right)=0$ for each $i$, and hence $D_{\gamma^{\prime}} v \in K(\gamma(s), t)$. As in the proof of Corollary 2.1 in [Ni and Tam 2003], we conclude that $w \in K(x, t)$. Since parallel translation preserves inner product, for each fixed $t$, the dimension of $K(x, t)$ is independent of $x$. We then use the de Rham decomposition theorem to conclude that a neighborhood of $p$ splits.

Note that $|\nabla f|^{2} \geq f$ on $U \times V$. In fact, for any $q \in V$, the restriction of $g$ and $f$ on $U \times\{q\}$ gives a soliton on $U \times\{q\}$ with zero Ric tensor. Lemma 2.1(2) implies that $\left|\nabla_{U \times\{q\}} f\right|^{2}=\left.f\right|_{U \times\{q\}}$, where $\nabla_{U \times\{q\}} f$ is the gradient of $\left.f\right|_{U \times\{q\}}$ with respect to the metric $\left.g\right|_{U \times\{q\}}$. Since $|\nabla f|^{2} \geq\left|\nabla_{U \times\{q\}} f\right|^{2}$, we infer that $|\nabla f|^{2}(x, q) \geq f(x, q)$ for all $x \in U$. Since $q$ is an arbitrary point in $V$, it follows that $|\nabla f|^{2} \geq f$ on $U \times V$.

We now prove that $|\nabla f|^{2} \leq f$ on $U \times V$. Given any point $y \in U \times V$, denote by $\gamma(s)$ the integral curve of $\nabla f /|\nabla f|^{2}$ such that $\gamma(0)=y$. Then $f(\gamma(s))=$ $s+f(\gamma(0))$. On the other hand, using Lemma 2.1(1) and (2), we have

$$
\begin{aligned}
\frac{d}{d s}|\nabla f|^{2}(\gamma(s)) & =\frac{1}{|\nabla f|^{2}} \nabla f\left(|\nabla f|^{2}\right)=\frac{1}{|\nabla f|^{2}}\left(|\nabla f|^{2}-\langle\nabla f, \nabla R\rangle\right) \\
& =\frac{1}{|\nabla f|^{2}}\left(|\nabla f|^{2}-2 \operatorname{Ric}(\nabla f, \nabla f)\right) .
\end{aligned}
$$


Since $\operatorname{Ric}(\nabla f, \nabla f) \geq 0$, we obtain $(d / d s)|\nabla f|^{2}(\gamma(s)) \leq 1$. Integrating this inequality from $-f(\gamma(0))$ to $s$ and noting that $\nabla f(\gamma(s))=0$ at $s=-f(\gamma(0))$ give us the desired inequality $|\nabla f|^{2} \leq f$.

We have thus proved that $|\nabla f|^{2}=f$, which, when combined with Lemma 2.1(2), implies that $R$ is constant in a neighborhood of $p$. Hence $R$ is constant on the entire $M$. The proof of Theorem 1.2 is therefore completed.

\section{Acknowledgements}

I thank Professors Peter Petersen and DaGang Yang for their interests in this work and for helpful discussions. I thank Professor Ovidiu Munteanu for pointing out an error in an earlier version of the paper. I also thank the referee for the thorough review and helpful suggestions.

\section{References}

[Bando 1987] S. Bando, "Real analyticity of solutions of Hamilton's equation", Math. Z. 195:1 (1987), 93-97. MR 88i:53073 Zbl 0606.58051

[Böhm and Wilking 2008] C. Böhm and B. Wilking, "Manifolds with positive curvature operators are space forms", Ann. of Math. (2) 167:3 (2008), 1079-1097. MR 2009h:53146 Zbl 1185.53073

[Cao 2007] X. Cao, "Compact gradient shrinking Ricci solitons with positive curvature operator", $J$. Geom. Anal. 17:3 (2007), 425-433. MR 2008h:53119 Zbl 1135.53044

[Cao and Zhou 2010] H.-D. Cao and D. Zhou, "On complete gradient shrinking Ricci solitons", J. Differential Geom. 85:2 (2010), 175-186. MR 2011k:53040 Zbl 1246.53051

[Cao et al. 2011] X. Cao, B. Wang, and Z. Zhang, "On locally conformally flat gradient shrinking Ricci solitons”, Commun. Contemp. Math. 13:2 (2011), 269-282. MR 2012e:53074 Zbl 1215.53061

[Chen 2009] B.-L. Chen, "Strong uniqueness of the Ricci flow", J. Differential Geom. 82:2 (2009), 363-382. MR 2010h:53095 Zbl 1177.53036

[Fernández-López and García-Río 2011] M. Fernández-López and E. García-Río, "Rigidity of shrinking Ricci solitons", Math. Z. 269:1-2 (2011), 461-466. MR 2012g:53072 Zbl 1226.53047

[Hamilton 1986] R. S. Hamilton, "Four-manifolds with positive curvature operator", J. Differential Geom. 24:2 (1986), 153-179. MR 87m:53055 Zbl 0628.53042

[Hamilton 1988] R. S. Hamilton, "The Ricci flow on surfaces", pp. 237-262 in Mathematics and general relativity (Santa Cruz, CA, 1986), edited by J. A. Isenberg, Contemp. Math. 71, Amer. Math. Soc., Providence, RI, 1988. MR 89i:53029 Zbl 0663.53031

[Hamilton 1995] R. S. Hamilton, "The formation of singularities in the Ricci flow", pp. 7-136 in Surveys in differential geometry, II (Cambridge, MA, 1993), edited by C. C. Hsiung and S.-T. Yau, Int. Press, Cambridge, MA, 1995. MR 97e:53075 Zbl 0867.53030

[Ivey 1993] T. Ivey, "Ricci solitons on compact three-manifolds", Differential Geom. Appl. 3:4 (1993), 301-307. MR 94j:53048 Zbl 0788.53034

[Kotschwar 2013] B. L. Kotschwar, "A local version of Bando's theorem on the real-analyticity of solutions to the Ricci flow", Bull. Lond. Math. Soc. 45:1 (2013), 153-158. MR 3033963 Zbl 1259.53065

[Morgan and Tian 2007] J. Morgan and G. Tian, Ricci flow and the Poincaré conjecture, Clay Mathematics Monographs 3, Amer. Math. Soc., Providence, RI, 2007. MR 2008d:57020 Zbl 1179.57045 
[Naber 2006] A. Naber, "Some geometry and analysis on Ricci solitons", preprint, 2006. arXiv math/0612532

[Ni and Tam 2003] L. Ni and L.-F. Tam, "Plurisubharmonic functions and the structure of complete Kähler manifolds with nonnegative curvature", J. Differential Geom. 64:3 (2003), 457-524. MR 2005a:32023 Zbl 1088.32013

[Ni and Wallach 2008] L. Ni and N. Wallach, "On a classification of gradient shrinking solitons", Math. Res. Lett. 15:5 (2008), 941-955. MR 2010i:53127 Zbl 1158.53052

[Perelman 2003] G. Perelman, "Ricci flow with surgery on three manifolds", preprint, 2003. arXiv math/0303109

[Petersen and Wylie 2009] P. Petersen and W. Wylie, "Rigidity of gradient Ricci solitons", Pacific J. Math. 241:2 (2009), 329-345. MR 2010j:53071 Zbl 1176.53048

[Petersen and Wylie 2010] P. Petersen and W. Wylie, "On the classification of gradient Ricci solitons", Geom. Topol. 14:4 (2010), 2277-2300. MR 2012a:53060 Zbl 1202.53049

Received March 21, 2014. Revised December 23, 2014.

Mingliang CAi

DEPARTMENT OF MATHEMATICS

UNIVERSITY OF MIAMI

Coral Gables, FL 33124

UNITED STATES

mcai@math.miami.edu 


\title{
PACIFIC JOURNAL OF MATHEMATICS
}

\author{
msp.org/pjm
}

Founded in 1951 by E. F. Beckenbach (1906-1982) and F. Wolf (1904-1989)

\section{EDITORS}

Don Blasius (Managing Editor)

Department of Mathematics

University of California

Los Angeles, CA 90095-1555

blasius@math.ucla.edu

\author{
Paul Balmer \\ Department of Mathematics \\ University of California \\ Los Angeles, CA 90095-1555 \\ balmer@math.ucla.edu \\ Robert Finn \\ Department of Mathematics \\ Stanford University \\ Stanford, CA 94305-2125 \\ finn@math.stanford.edu \\ Sorin Popa \\ Department of Mathematics \\ University of California \\ Los Angeles, CA 90095-1555 \\ popa@math.ucla.edu
}

\author{
Vyjayanthi Chari \\ Department of Mathematics \\ University of California \\ Riverside, CA 92521-0135 \\ chari@math.ucr.edu \\ Kefeng Liu \\ Department of Mathematics \\ University of California \\ Los Angeles, CA 90095-1555 \\ liu@math.ucla.edu \\ Jie Qing \\ Department of Mathematics \\ University of California \\ Santa Cruz, CA 95064 \\ qing@ cats.ucsc.edu
}

\section{PRODUCTION}

Silvio Levy, Scientific Editor, production@msp.org

\section{SUPPORTING INSTITUTIONS}

ACADEMIA SINICA, TAIPEI

CALIFORNIA INST. OF TECHNOLOGY

INST. DE MATEMÁTICA PURA E APLICADA

KEIO UNIVERSITY

MATH. SCIENCES RESEARCH INSTITUTE

NEW MEXICO STATE UNIV.

OREGON STATE UNIV.

\author{
STANFORD UNIVERSITY \\ UNIV. OF BRITISH COLUMBIA \\ UNIV. OF CALIFORNIA, BERKELEY \\ UNIV. OF CALIFORNIA, DAVIS \\ UNIV. OF CALIFORNIA, LOS ANGELES \\ UNIV. OF CALIFORNIA, RIVERSIDE \\ UNIV. OF CALIFORNIA, SAN DIEGO \\ UNIV. OF CALIF., SANTA BARBARA
}

\author{
Daryl Cooper \\ Department of Mathematics \\ University of California \\ Santa Barbara, CA 93106-3080 \\ cooper@math.ucsb.edu \\ Jiang-Hua Lu \\ Department of Mathematics \\ The University of Hong Kong \\ Pokfulam Rd., Hong Kong \\ jhlu@maths.hku.hk \\ Paul Yang \\ Department of Mathematics \\ Princeton University \\ Princeton NJ 08544-1000 \\ yang@math.princeton.edu
}

These supporting institutions contribute to the cost of publication of this Journal, but they are not owners or publishers and have no responsibility for its contents or policies.

See inside back cover or msp.org/pjm for submission instructions.

The subscription price for 2015 is US \$420/year for the electronic version, and \$570/year for print and electronic.

Subscriptions, requests for back issues and changes of subscribers address should be sent to Pacific Journal of Mathematics, P.O. Box 4163, Berkeley, CA 94704-0163, U.S.A. The Pacific Journal of Mathematics is indexed by Mathematical Reviews, Zentralblatt MATH, PASCAL CNRS Index, Referativnyi Zhurnal, Current Mathematical Publications and Web of Knowledge (Science Citation Index).

The Pacific Journal of Mathematics (ISSN 0030-8730) at the University of California, c/o Department of Mathematics, 798 Evans Hall \#3840, Berkeley, CA 94720-3840, is published twelve times a year. Periodical rate postage paid at Berkeley, CA 94704, and additional mailing offices. POSTMASTER: send address changes to Pacific Journal of Mathematics, P.O. Box 4163, Berkeley, CA 94704-0163.

PJM peer review and production are managed by EditFLOW ${ }^{\circledR}$ from Mathematical Sciences Publishers.

\section{PUBLISHED BY}

\section{mathematical sciences publishers \\ nonprofit scientific publishing}

http://msp.org/

(C) 2015 Mathematical Sciences Publishers 


\section{PACIFIC JOURNAL OF MATHEMATICS}

Volume $277 \quad$ No. $1 \quad$ September 2015

Real positivity and approximate identities in Banach algebras

DAVID P. BLECHER and NARUTAKA OZAWA

On shrinking gradient Ricci solitons with nonnegative sectional curvature 61

Mingliang CAI

From quasimodes to resonances: exponentially decaying perturbations

ORAN GANNOT

A general simple relative trace formula

JAYCE R. GETZ and HEEKYOUNG HAHN

Chern-Simons functions on toric Calabi-Yau threefolds and

Donaldson-Thomas theory

\section{ZHENG HUA}

On the flag curvature of a class of Finsler metrics produced by the navigation problem

LiBING HUANG and XIAOHUAN MO

Angular distribution of diameters for spheres and rays for planes

NOBUHIRO INNAMI and YUYA UNEME

A note on an $L^{p}$-Brunn-Minkowski inequality for convex measures in the 187 unconditional case

ARNAUD MARSIGLIETTI

Structure of seeds in generalized cluster algebras

TOMOKI NAKANISHI

Inequalities of Alexandrov-Fenchel type for convex hypersurfaces in hyperbolic space and in the sphere

Yong WeI and ChangWei XiONG

Upper bounds of root discriminant lower bounds 